\title{
CONTRIBUIÇÕES TEÓRICAS E CONCEITUAIS DE VIGOTSKI PARA A PESQUISA QUALITATIVA EM EDUCAÇÃ̃ ${ }^{i}$
}

VYGOTSKY'S THEORETICAL AND CONCEPTUAL CONTRIBUTIONS TO QUALITATIVE RESEARCH IN EDUCATION

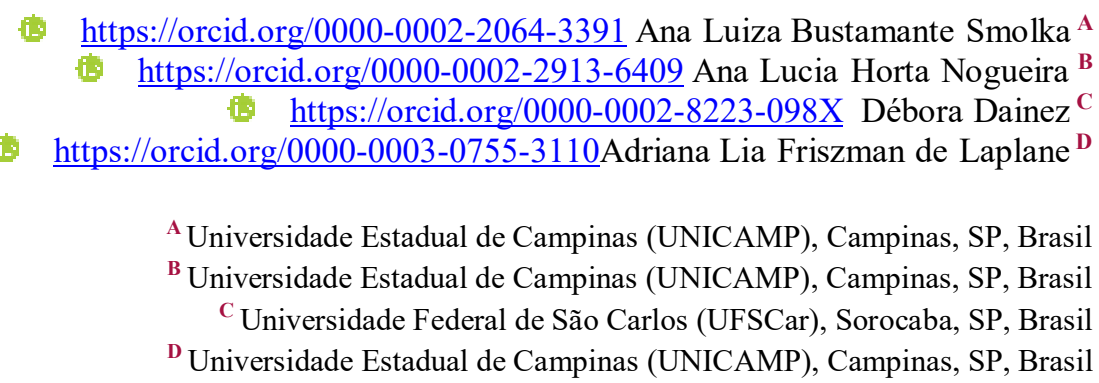

Recebido em: 13 ago. 2021 | Aceito em: 15 ago. 2021 Correspondência: Ana Smolka (asmolka@unicamp.br)

\section{Resumo}

A abordagem de Vigotski para o desenvolvimento humano está profundamente entrelaçada com sua investigação metodológica. Essa investigação está relacionada à sua persistente busca por compreender o problema da consciência. A incansável busca do autor por uma explicação plausível das bases materiais das funções psíquicas especificamente humanas permeia seu trabalho teórico, prático e empírico nos campos da psicologia e da educação. Nessa sua busca, a sociogênese e a mediação semiótica, tomadas inicialmente como hipóteses investigativas, tornam-se princípios explicativos. Trechos de seus textos seminais permitem acompanhar a elaboração dos pressupostos epistemológicos que ancoram sua teorização e evidenciam as relações entre objeto de estudo, princípio explicativo e unidade de análise nas pesquisas do desenvolvimento cultural do ser humano. Uma de suas principais preocupações dizia respeito aos modos de ensinar e de estudar as relações de ensino, bem como aos resultados ou efeitos dessas relações. Falar sobre as elaborações teóricas de Vigotski é, portanto, falar sobre o método - de inquirir, de investigar, de ensinar. Do início ao fim de seu esforço teórico, encontramos uma profunda preocupação sobre o que significa ser humano e quais são os meios/modos de se tornar humano. As repercussões e contribuições da abordagem de Vigotski para a pesquisa em educação, assim como suas implicações éticas e políticas, merecem ser destacadas. Sua forma de conceber o método escapa da rigidez, não do rigor, apontando para uma instigante flexibilidade que aproxima seus esforços aos de muitos autores contemporâneos em diferentes campos de conhecimento.

Palavras-chave: Vigotski; método; pesquisa em educação; desenvolvimento cultural, sociogênese; mediação semiótica; perspectiva histórico-cultural.

\begin{abstract}
Vygotsky's approach to human development is profoundly intertwined with his methodological inquiry. This inquiry is related to his persistent quest for framing and understanding the problem of consciousness. His untiring search for a plausible explanation of the material basis of specifically human psychological functions pervades his theoretical, practical, and empirical work in the fields of psychology and education. Throughout this search,
\end{abstract}


sociogenesis and semiotic mediation, at first investigative hypotheses, become explanatoryprinciples. Excerpts from his seminal texts allow us to follow the elaboration of epistemological assumptions that anchor his process of theorization and evidence the interrelationships between object of study, explanatory principle, and unit of analysis in studies of cultural development. One of his major concerns had to do with the ways of teaching and the ways of studying teaching relations, as well as the results or effects of such relations. To talk about Vygotsky's theoretical elaborations is, hence, to talk about method - of inquiring, of studying, of teaching. From the beginning through to the end of his theoretical endeavor, we find a deep concern about what it means to be human, what are the means to be human. Repercussions and contributions of Vygotsky's approach to research in education, as well as their ethical and political implications, must be highlighted. His way of conceiving method escapes from rigidity, not from rigor, pointing to aninstigating flexibility which approximates his efforts to the efforts of many contemporary authors in different fields.

Keywords: Vygotsky; method; research in education; cultural development; sociogenesis; semiotic mediation; cultural-historical approach.

\section{Introdução}

Os trabalhos teóricos e empíricos de L. S. Vigotski, realizados nas intersecções dos campos da psicologia e da educação nas primeiras décadas do século XX, consistem numa tentativa genuína de estudar o desenvolvimento humano em movimento, no seu processo inerente de constituição e (trans)formação histórica. Vigotski concebia o desenvolvimento cultural do indivíduo como uma história pessoal intrinsecamente tecida nas práticas culturais e na história humana. Uma das questões persistentes que permearam suas obras foram os modos de indagar e investigar a emergência de novas possibilidades e formas de ação humana, no entrelaçamento da ontogênese, da cultura e da história.

Ao longo de seu trabalho investigativo, ao abordar uma diversidade de temas relacionados à conduta e à consciência humana, Vigotski elaborou diversos procedimentos metodológicos e analíticos. Ele argumentou de forma consistente sobre as relações intrínsecas entre o método de pesquisa e o problema sob investigação, defendendo veementemente a necessidade de se buscar um método adequado às possíveis configurações de um objeto de estudo.

Para dar visibilidade ao engendramento de novas formas de ação e funcionamento mental, o autor propôs o que chamou de método instrumental, de estimulação dupla, ou histórico-genético, e argumentou sobre a importância de se desenvolverem projetos experimentais relacionados ao trabalho pedagógico. De fato, uma de suas maiores preocupações dizia respeito aos modos de ensinar e aos modos de investigar as relações de ensino, bem como aos resultados ou efeitos de tais relações. 
A questão do método está no cerne da proposta de Vigotski. Toda a sua obra é permeada por uma investigação metodológica. Ao abordarmos seus estudos sobre o desenvolvimento humano, destacamos seus fundamentos históricos e dialéticos e enfatizamos a dimensão éticopolítica da produção do conhecimento, destacando assim o compromisso social inscrito no próprio ato de pesquisar. Sob esse prisma, as formas de levantar os problemas e colocar as questões na pesquisa educacional, de projetar e conduzir estudos que provoquem desenvolvimento, já expressam um compromisso social.

Particularmente no campo da educação, essa perspectiva teórico-metodológica torna-se fecunda na ancoragem a trabalhos de pesquisa que vinculem a problemática geral do desenvolvimento humano às práticas sociais, políticas e educacionais, abrindo novas possibilidades de atividade e práxis humanas. Torna-se, assim, possível contribuir para a investigação do desenvolvimento humano e da vida social na medida em que efetivamente colocamos em perspectiva as práticas educativas (trans)formadoras.

No presente texto, nossos objetivos são: (1) contextualizar a obra do autor no ambiente histórico e cultural de sua época; (2) explicitar suas principais preocupações e os pressupostos básicos que orientam sua busca pela compreensão do desenvolvimento humano; (3) discutir sua concepção de atividade investigativa e a elaboração de métodos de pesquisa nos campos da psicologia e da educação; (4) explorar as implicações de sua abordagem para a pesquisa contemporânea em educação.

\section{Vigotski e o ambiente histórico-cultural de seu tempo}

As ideias de Lev Semionovitch Vigotski, elaboradas nas primeiras décadas do século XX, foram amplamente difundidas, principalmente a partir do início dos anos 1980, e vêm conquistando adeptos em todo o mundo, impactando as formas de fazer pesquisa na área da educação.

Todas as biografias de Vigotski mencionam a extensão de sua obra em relação à brevidade de sua vida. Nascido na Bielo-Rússia, em 1896, segundo filho de família judia, Vigotski morreu cedo, aos 37 anos, e deixou um conjunto de ideias inspiradoras ao trabalhar nas interseções de várias áreas do conhecimento, como os estudos da linguagem e literatura, psicologia, filosofia, antropologia, sociologia, neurologia.

Imerso em um rico ambiente cultural, Vigotski viveu em meio a uma enorme efervescência de ideias. Impactado pelas demandas e propostas da Revolução Russa, sentiu profundamente as condições e as contradições das relações sociais. Vivenciou um período de rápida industrialização e modernização dos meios de produção, concomitantemente a uma 
intensa produção artística e científica. Profundas diferenças entre as condições de vida urbana e agrária tornaram-se bastante visíveis e acentuadas. Participando desse momento histórico, Vigotski sofreu discriminação em razão de suas raízes judaicas e tomou consciência das profundas desigualdades sociais. Compartilhando o ideal revolucionário de uma emergente e engajada juventude, ele aderiu à utopia da construção do "homem novo". Trabalhando como professor de literatura e atuando como crítico de artes na pequena cidade de Gomel, tornou-se um famoso psicólogo e pesquisador do desenvolvimento humano, genuinamente preocupado e comprometido com o processo educacional.

Atento aos problemas e controvérsias de sua época, Vigotski conheceu, examinou e debateu as mais diversas ideias de diferentes campos do saber. Abordou temas como as formas e funções da linguagem; a criação literária e a função poética; a reação estética; evolução e revolução; hereditariedade e meio ambiente; consciência e comportamento; história e desenvolvimento; idealismo e materialismo; o status da ciência; transformações sociais, entre outros.

Apaixonado por literatura e arte, ele tinha 19 anos quando escreveu a primeira versão de seu estudo sobre $A$ tragédia de Hamlet (VYGOTSKI, 1999a), que mais tarde revisitaria e revisaria, para integrar seu trabalho de doutorado em Psicologia da arte (VIGOTSKI, 1999b). Nessa mesma época, escreveu sobre ensino e educação escolar em uma coleção de textos que veio a compor a Psicologia Pedagógica (VIGOTSKI, 2001a) ${ }^{1}$.

Para ele, A tragédia de Hamlet sintetiza o drama humano. É esse drama, vivido na dimensão social e subjetiva, que se entrelaça às condições concretas da vida de Vigotski. Além de viver a ocupação alemã na Rússia, durante a qual dois de seus irmãos morreram, Vigotski assumiu os cuidados de sua mãe e de outro irmão com tuberculose, que posteriormente também faleceu. O jovem leitor de Hamlet também contraiu tuberculose. A consciência da iminência da morte deu origem a um sentimento trágico. Viver sob a iminência da morte, em uma era revolucionária, tornava alguns projetos de vida urgentes e inevitáveis.

Os últimos dez anos de sua vida - 1924 a 1934 - concentram a sua intensa produção. O crítico literário, que se tornou professor, também se tornou pesquisador do drama humano. Com Alexander Luria e Alexei Leontiev, seus companheiros de trabalho mais próximos, Vigotski compôs um grupo de pesquisa na Universidade de Moscou, conhecido como "troika" na psicologia russa.

1 A tragédia de Hamlet foi publicada pela primeira vez em 1915; Psychology of Art em 1925; Educational Psychology em 1926. 
Trabalhando no Laboratório de Defectologia, criado em 1925, que recebia crianças com patologias diversas e desenvolvimento atípico, Vigotski defendeu e propôs diversas formas de atuar pedagogicamente e de realizar pesquisas sobre o desenvolvimento humano, questionando as condições e possibilidades da atividade humana criadora.

Literatura e arte, pedagogia (orientações e modos de ensinar), pedologia (a ciência do desenvolvimento infantil), psicologia (o estudo do desenvolvimento humano e da consciência na história e cultura) e defectologia articulam-se no campo de interesse e pesquisa de Vigotski.

A noção de drama, inicialmente ancorada na obra de Shakespeare, foi redimensionada nos estudos de Vigotski e no diálogo com as contribuições de muitos outros autores: a dialética de Hegel; a noção de socius de Pierre Janet; o monismo de Spinoza e sua ideia de affectus; as distintas noções de catarse de Aristóteles e Freud; a teoria da evolução de Darwin; os trabalhos de Pavlov sobre os reflexos e o sistema nervoso; os estudos da Gestalt sobre percepção; a teoria de campo de Lewin; o drama da vida cotidiana em Politzer; e, mais fortemente, a filosofia de Marx e o materialismo histórico-dialético.

Nesse movimento de busca e indagação, a noção de drama adquiriu um novo status: foi concebido como um modo de organização e funcionamento do psiquismo humano, uma característica da consciência historicamente elaborada. No cerne dessa consciência dramática, Vigotski enfatizou a forma verbal da linguagem que, como formação histórica, não só possibilitou o trabalho simbólico compartilhado "além da pele", mas possibilitou a cada ser humano experimentar na esfera privada as funções, tensões, e contradições das relações sociais.

A vivência desse drama no nível pessoal e a consciência desse drama no nível histórico levaram Vigotski a refletir sobre o inevitável determinismo do mundo material em relação às determinações históricas decorrentes da atividade humana e as possibilidades de construção da vontade, ou determinação, na esfera individual. Sua vida e obra evidenciam sua tese: a constituição social da personalidade individual e as condições de possibilidades de protagonismo do sujeito, forjadas na história das relações sociais.

\section{O problema da consciência e a busca por uma psicologia concreta}

Para compreender a proposta metodológica de Vigotski e o estatuto do método em sua teoria, é fundamental conhecer as principais preocupações e os pressupostos básicos que orientam sua investigação sobre o desenvolvimento humano. Alguns dos textos iniciais do autor podem nos dar um vislumbre de suas preocupações e dos esforços contínuos que marcam a história de sua obra: "Temos que falar abertamente. Os enigmas da consciência, os enigmas da mente não podem ser evitados com truques metodológicos ou subterfúgios de princípio" Revista Interinstitucional Artes de Educar. Rio de Janeiro, V.7, N.3 - pág.1364-1389 set-dez de 2021: "Dossiê Relatos de experiências e produção acadêmica”- DOI: https://doi.org/10.12957/riae.2021.63920 
(VIGOTSKI, 1994, p. 41; tradução nossa). "A consciência é a vivência das vivências [...] O ser determina a consciência. (VIGOTSKI, 1996, p. 71).

O que se pode buscar nos mestres do marxismo de antemão não é uma solução para a questão, nem mesmo uma hipótese de trabalho [...] mas o método para desenvolvêla. Não quero aprender o que constitui a mente de graça, escolhendo algumas citações, quero aprender de todo o método de Marx como construir uma ciência, como abordar a investigação da mente. (VIGOTSKI, 1996, p. 395).

NB A palavra história (psicologia histórica) para mim significa duas coisas: 1) abordagem dialética geral das coisas - neste sentido qualquer coisa tem sua história, neste sentido Marx: uma ciência - a história (Arquivo, p. X), ciências naturais = história da natureza, história natural; 2) história no próprio sentido, isto é a história humana. Primeira história $=$ materialismo dialético, a segunda - materialismo histórico. As funções superiores diferentemente das inferiores, no seu desenvolvimento, são subordinadas às regularidades históricas (veja o caráter dos gregos e o nosso). Toda a peculiaridade do psiquismo do homem está em que nele são unidas (síntese) uma e outra história (evolução + história). O mesmo no desenvolvimento infantil (compare as duas linhas). (VIGOTSKI, 2000, p. 23).

O conjunto de citações acima pode ser tomado como indicativo da busca do autor pelo que ele denomina psicologia geral, científica, humana e concreta (VIGOTSKI, 2000, 1996). Por meio da leitura desses textos, podemos rastrear as análises e questionamentos de Vigotski sobre as tendências psicológicas de sua época e acompanhar o seu processo simultâneo de apropriação do materialismo histórico-dialético. Em diálogo com as teorias psicológicas da época - cujas contribuições destaca e às quais adere parcialmente -, ele critica fortemente as formas estabelecidas de abordagem do comportamento humano, apontando para os limites das visões dualistas, sejam de posições idealistas, subjetivistas ou introspeccionistas, que consideravam os fenômenos psíquicos imateriais; ou posições organicistas que reduziam a explicação do comportamento humano a reações, reflexos condicionados e à utilidade biológica da psique.

Em sua famosa palestra na Universidade de Moscou em 1924, intitulada “A consciência como problema da psicologia do comportamento", Vigotski discutiu três maneiras de conceber, hipotetizar e estudar o comportamento humano, enfatizando a importância de colocar a consciência como um objeto de investigação relevante e acessível: a consciência como reflexo dos reflexos, a consciência como questão da estrutura do comportamento e a consciência como característica da atividade laboral humana (DAVYDOV; RADZIKHOVSKII, 1985; VERESOV, 1999; VIGOTSKI, 1994). Afirmando as inescapáveis condições materiais, biológicas e orgânicas da vida humana, ele argumentou insistentemente que, além dos reflexos, a estrutura do comportamento humano deveria ser considerada; e, ao considerar o comportamento humano, a vontade e a consciência, como características humanas específicas, 
deveriam ser levadas em consideração. A questão era como abordar o problema e como propor o método adequado e os procedimentos analíticos.

A reconhecida relevância das contribuições de Vigotski nessa palestra pode ser vista na crítica às tendências psicológicas da época; em suas reflexões sobre a experiência humana social, histórica, duplicada, mediada por palavras e signos; em sua ênfase no conceito de atividade (atividade laboral prática) como a realidade que determina a mente; na distinção que ele faz entre objeto de estudo, unidade de análise e princípio explicativo na investigação psicológica da consciência (DAVYDOV; RADIZKHOVSKII, 1985).

Nessa linha de raciocínio observa-se o empenho de Vigotski em sua busca por um princípio explicativo para a consciência humana, no seu diálogo incansável com reflexologistas, behavioristas, educadores, linguistas, artistas e a filosofia de Marx. Sua abordagem foi uma alternativa tanto à reflexologia clássica quanto ao behaviorismo clássico (VERESOV, 1999). O que ele propôs nesta palestra foi uma tentativa ousada de mudar o foco e a perspectiva nos estudos psicológicos; uma tentativa que ganharia força e consistência interna em seus estudos posteriores.

Nas notas que compõem o "Manuscrito de 1929”, ou Psicologia Concreta (VIGOTSKI, 2000), Vigotski discute o problema das complexas e contraditórias relações entre natureza/cultura, referindo-se à dialética da natureza e da história humana (MARX, 1999; MARX; ENGELS, 1987). A natureza em si não tem história, mas produz as condições para o surgimento do Homo sapiens. Nesse processo emergente, a espécie Homo torna-se capaz de transformar a natureza por meio da atividade coletiva, pela criação de ferramentas e instrumentos, e passa a produzir suas próprias condições de existência, a produzir cultura e história, (trans)formando-se neste processo de produção. $\mathrm{O}$ confronto coletivo e a superação das necessidades básicas para a sobrevivência das espécies abrem novas possibilidades de transformação da natureza e de elaboração do conhecimento, viabilizando a emergência histórica da consciência nas condições concretas e materiais da vida. A história do homem é, portanto, a história da transformação do seu próprio modo de ser, o que implica o percurso da ordem da natureza à ordem da cultura (PINO, 2000). O ser humano torna-se o artífice, o arquiteto de si mesmo.

Vigotski buscou entender - e encontrar uma explicação plausível - para o desenvolvimento cultural do ser humano nesta história de produção de sua própria existência. É sob o prisma da história e da dialética que o autor aborda e estuda o comportamento e o desenvolvimento humano. Como Leontiev admitiu, a questão da metodologia é a principal questão "quando estamos lidando com o trabalho criativo de Vigotski". A dialética interna, 
assim como a perspectiva histórica no exame de um fenômeno, formava, em princípio, o traço característico de seu pensamento. "A compreensão dos fundamentos da dialética marxista elevou o pensamento de Vigotski a um novo nível qualitativo" (LEONTIEV, 1997, p. 19; tradução nossa).

De fato, quando voltamos aos seus primeiros textos (1921-1924), encontramos mais elementos para compor uma história das elaborações teóricas de Vigotski relacionadas à sua prática como professor e pesquisador em educação.

Na abertura de sua Psicologia Pedagógica, Vigotski se indaga sobre o trabalho pedagógico e considera que o problema da educação está no cerne da nova psicologia. Explicita, ainda, que a sua principal intenção é a construção de um sistema científico objetivo e rigoroso de psicologia educacional (VIGOTSKI, 2001a). Nesta coletânea de textos, em que aborda um amplo espectro de questões educacionais, ele admite claramente o papel e a importância do meio social, afirmando que "O homem não entra em convívio com a natureza senão através do meio (social), e em função disso, o meio se torna o fator mais importante que determina e estabelece o comportamento do homem” (VIGOTSKI, 2001a, p. 6-7).

Nesse momento de elaboração de Vigotski a importância do meio ambiente como fator determinante do comportamento humano parecia permanecer a meio caminho entre a abordagem behaviorista e os princípios marxistas. Apesar de se referir às ideias de Marx e Engels, assumindo o projeto de um novo homem, alegando que a revolução ocupa-se da reeducação de toda a humanidade (VAN DER VEER; VALSINER, 1991) e falando em uma sociedade sem classes, sua maneira de explicar "objetivamente" o impacto do meio ambiente estava ancorada na natureza e na estrutura dos estímulos que moldam a criança ou aos quais ela reage. A noção de ambiente como algo externo ao indivíduo, como um fator interveniente e socialmente determinante, ainda prevalece nos argumentos de Vigotski sobre o comportamento humano.

Dois outros campos de envolvimento e ação, relacionados ao trabalho prático e empírico de Vigotski neste mesmo período, também contribuem para suas elaborações conceituais e teóricas: seus estudos em Psicologia da arte (1999b) e sua experiência no campo da defectologia (1925-1929), dois campos aparentemente contrastantes que constituem, para Vigotski, locus específico de profunda preocupação com a condição, experiência e consciência humanas.

Em Psicologia da arte, uma questão central era: Como uma obra de arte, mais especificamente um texto literário, pode provocar uma reação estética? Qual seria a natureza desse fenômeno humano? Como estudá-lo? - questões que provocaram intenso debate entre 
formalistas, linguistas, críticos literários. Para Vigotski, a teoria do materialismo histórico, que busca construir uma análise científica da arte com base nos mesmos princípios aplicados ao estudo de todas as formas e fenômenos da vida social, parecia ser a tendência investigativa mais avançada e coerente (VIGOTSKI, 1999b). Ao admitir que os estudos em psicologia tinham se concentrado principalmente no trabalho do artista ou na reação do receptor, ele propôs que a psicologia concentrasse sua análise na obra de arte em si. Inspirado pela maneira de investigar do historiador, sugeriu a necessidade de introduzir métodos analíticos indiretos adequados à criação de um novo objeto de estudo.

Ao mesmo tempo, Vigotski estava intensamente envolvido em seu trabalho com crianças com deficiência, perguntando-se sobre as condições de educação e as possibilidades de conhecimento, vontade e consciência nos mais diversos e graves casos de deficiência física e/ou mental.

Suas primeiras abordagens nesse campo basearam-se na reflexologia e no behaviorismo, teorias que lhe deram os recursos iniciais para trabalhar e estudar "objetivamente" tais casos. No entanto, os limites teóricos e práticos de ambas as abordagens teóricas tornaram-se bastante evidentes, impelindo-o a buscar princípios explicativos mais consistentes e levando-o a aprofundar a teorização histórico-cultural. Esse foi o maior desafio que ele enfrentou em sua busca e argumentos para uma teoria geral do desenvolvimento humano (KOZULIN, 1990; VIGOTSKI, 1997; VAN DER VEER; VALSINER, 1991; VERESOV, 1999; VIGOTSKI, 2018).

O estudo do desenvolvimento de crianças com diferentes deficiências o levou a se opor fortemente a abordagens quantitativas e a defender a necessidade de considerar a qualidade, a diversidade e a singularidade dos processos de desenvolvimento. Assim, defendeu um estudo profundo e abrangente dessa diversidade e uma abordagem atenta e cuidadosa dos períodos e transformações do desenvolvimento (VIGOTSKI, 1997).

O trabalho de Vigotski no campo da educação, particularmente no campo da defectologia, seu amplo e profundo conhecimento de teorias em psicologia, sua experiência e estudos sobre arte e literatura, seu diálogo com o materialismo histórico e dialético de Marx e Engels, contribuíram para sua atenção especial ao papel mediador e à função dos instrumentos e signos na atividade humana, bem como ao estatuto e poder da palavra, a forma verbal da linguagem no desenvolvimento humano e na consciência. O levantamento de algumas hipóteses - da natureza social do psiquismo humano, do signo como instrumento psicológico, da atividade mediada, da internalização da cultura - surgiu como resultado de suas experiências 
vividas e sua investigação filosófica e metodológica a respeito das condições concretas da existência humana.

\section{Das hipóteses aos princípios: desenvolvendo métodos de pesquisa}

Conforme Vigotski incorporava e reelaborava as teses, suposições e argumentos da filosofia marxista, passou a defender e a postular as origens sociais ou a sociogênese do desenvolvimento humano, afirmando a primazia das relações sociais no desenvolvimento ontogenético. "Não a natureza, mas a sociedade deve, em primeiro lugar, ser considerada um fator determinante do comportamento humano. Essa é a ideia de desenvolvimento cultural da criança" (VIGOTSKI, 1995, p. 89, nossa tradução). A noção de ambiente como meio social não implica simplesmente uma circunstância contextual impactando e modelando o comportamento da criança, mas envolve a concepção das relações sociais como um princípio explicativo na formação do psiquismo individual.

No entanto, assumir que o indivíduo é social em suas origens e modos de ser ainda não resolve problemas empíricos e não responde a questões teóricas. A questão persistia: como estudar o desenvolvimento e o comportamento humano? Como ter acesso à consciência? Como abordar as relações entre indivíduo e sociedade a partir de uma perspectiva psicológica? E como conceber e acessar o psiquismo humano em sua unidade ou totalidade dinâmica e complexa?

Uma hipótese inicial de mediação por instrumentos e signos na atividade humana, tanto na dimensão histórica quanto individual, levou Vigotski a propor e desenvolver métodos de estudo que pudessem dar visibilidade ao engendramento de novas formas de ação e ao surgimento de modos novos ou únicos de operação. Essa hipótese deu origem ao que chamou de método instrumental, histórico-genético ou de dupla estimulação, por meio do qual buscou estudar a gênese dos processos, visando criar condições para provocar o desenvolvimento e evidenciar aspectos da dinâmica social/individual do funcionamento psíquico. Os grandes desafios eram como conceber, como interpretar e como dar visibilidade a processos ocultos, não imediatamente observáveis.

Tomando como ponto de partida que a atividade humana era mediada por instrumentos e signos criados pelo homem, e que essa atividade mediada integra a base material do funcionamento psíquico, a questão era como evidenciar os modos de produzir, apropriar-se e transformar meios e modos da ação humana no curso da história. Diante desse problema, Vigotski propôs "O método instrumental em psicologia" (1996), afirmando que: "Por sua própria essência, o método instrumental é um método histórico-genético. Ele introduz um ponto de vista histórico na investigação do comportamento: o comportamento pode ser entendido 
apenas como a história do comportamento (Blonsky)" (VIGOTSKI, 1996, p. 98). Conceber o desenvolvimento ontogenético intrinsecamente entrelaçado ao movimento histórico e projetar situações experimentais para estudar tal desenvolvimento foi um gesto importante e audacioso. Ele comenta alguns dos resultados da pesquisa:

\begin{abstract}
Quando estudamos os processos das funções superiores em crianças, chegamos à seguinte conclusão surpreendente: cada forma superior de comportamento entra em cena duas vezes em seu desenvolvimento - primeiro como uma forma coletiva de comportamento, como uma função interpsicológica, depois como uma função intrapsicológica, como uma determinada maneira de se comportar. Não percebemos esse fato porque é muito comum e, portanto, estamos cegos a ele. $\mathrm{O}$ exemplo mais marcante é a fala. A princípio a fala é um meio de contato entre a criança e as pessoas ao seu redor, mas quando a criança começa a falar para si mesma, isso pode ser considerado a transferência de uma forma coletiva de comportamento para a prática do comportamento pessoal. (VIGOTSKI, 1996, p. 112).
\end{abstract}

Os comentários de Vigotski sobre as conclusões de sua pesquisa apontam para algumas questões metodológicas relevantes: (1) Como os processos de funções superiores em crianças se configuram como objeto de estudo? Como são estudados empiricamente? (2) O que se torna perceptível? Como? O que pode ser "visto" no desenvolvimento de um trabalho de pesquisa? (3) Como as formas de conceber afetam o que é ou pode ser visto em um trabalho empírico?

As análises de situações prototípicas exemplares nos estudos dos processos de memorização, discurso egocêntrico, escrita inicial, brincadeira infantil, formação de conceitos e zona de desenvolvimento proximal podem ser acompanhadas e apreciadas em vários relatos e relatórios de pesquisa (LEONTIEV, 2009; LURIA, 1977; VIGOTSKI, 1984, 2001b; VIGOTSKI; LURIA, 1994). Em todos os casos, Vigotski e seus colaboradores parecem estar atentos e cuidadosos quanto às possíveis formas de proceder através do projeto e da análise de situações experimentais. Em todos os casos, podemos ver reiterados os princípios metodológicos propostos: (1) estudar processos, e não objetos estáticos; (2) buscar explicações de tais processos, não trabalhar com meras descrições; (3) buscar a essência do fenômeno, além da aparência superficial; (4) estudar os processos em movimento, ou seja, provocar mudança e desenvolvimento. Subjacente a essa proposta metodológica estava uma ênfase na abordagem histórico-genética e na função mediadora dos instrumentos psicológicos, que constituíam, na época, uma forma totalmente nova de investigação (VIGOTSKI, 1984; 1996; VIGOTSKI; LURIA, 1994).

Os resultados do trabalho de pesquisa dariam suporte à formulação posterior da lei genética geral do desenvolvimento humano (VIGOTSKI, 1996). As hipóteses iniciais deram lugar à afirmação de teses, transformaram-se em princípios. Essas formas teóricas de conceber dialeticamente afetaram as formas de ver e interpretar os fenômenos empíricos. 
Ao reconhecer o papel dos signos no desenvolvimento histórico das funções psíquicas, Vigotski enxergou na possibilidade humana de significação - ou seja, na condição de produção de signos e sentidos nas relações interpessoais - uma forma plausível de compreensão dos meios/modos de internalização da cultura pela criança. A interpretação da gênese ou emergência do gesto de apontar na relação adulto-criança-objeto é indicativa do papel dos signos na constituição do funcionamento psíquico; contribui para a explicação de como as relações sociais internalizadas tornam-se funções psíquicas superiores (VIGOTSKI, 1995; 2000). O papel do signo no funcionamento psicológico adquire novo status nas teorizações de Vigotski:

É também notável que os signos, cujo significado nos parece tão grande na história do desenvolvimento cultural (como é demonstrado pela história de seu desenvolvimento), originalmente formem meios de contato, meios de agir sobre os outros. Quando consideramos sua origem real, cada signo é um meio de contato e poderíamos dizer mais amplamente que é um meio de contato entre certas funções mentais de caráter social. Transferido para o self, é também um meio de combinar funções na pessoa e seremos capazes de demonstrar que sem o signo o cérebro e as conexões originais não podem formar relações tão complexas quanto podem em razão da fala. (VIGOTSKI, 1996, p. 11).

Consequentemente, Vigotski encontra na possibilidade humana de produção de signos um princípio explicativo para o funcionamento do psiquismo humano, social e individual. Ele admite que o signo transforma as relações interfuncionais. Signos, palavras, conceitos são os instrumentos psicológicos mediadores, não orgânicos, socialmente produzidos, que tornam possível a inter/intra-regulação da atividade humana. Ele afirma: "A sociogênese é a chave para a conduta superior. Aqui nós ncontramos a função psicológica da palavra” (VIGOTSKI, 2000, p. 31). Mas o que significa esse termo psicológico? "De onde vem a função volitiva da palavra para nós, por que a palavra subordina a si as reações motoras? De onde vem o poder da palavra sobre a conduta?" (VIGOTSKI, 2000, p. 25).

De fato, essas questões levantadas no artigo de 1929 (VIGOTSKI, 2000) constituem uma linha fundamental da pesquisa e investigação de Vigotski, levando a seu trabalho final sobre Pensamento e linguagem (VIGOTSKI, 2001b), cujo prefácio e capítulo 1 soam como uma aula magna sobre método. Na tentativa de se apropriar e trabalhar metodologicamente a ideia de totalidade de Marx e a forma de conceber as relações todo/parte, Vigotski começa explicando:

\footnotetext{
“A psicologia que deseje estudar as unidades complexas precisa entender isso. Deve substituir o método de decomposição em elementos pelo método de análise que desmembra em unidades. Deve encontrar essas propriedades que não se decompõem e se conservam, são inerentes a uma dada totalidade enquanto unidade, e descobrir aquelas unidades em que essas propriedades estão representadas num aspecto contrário para, através dessa análise, tentar resolver as questões que se lhe apresentam.
} 
Que unidade é essa que se deixa decompor e contém propriedades inerentes ao pensamento verbalizado como uma totalidade? Achamos que essa unidade pode ser encontrada no aspecto interno da palavra: no seu significado". (VIGOTSKI, 2021, p.8).

Estabelecendo inicialmente uma analogia com uma gota d'água, que mantém a composição unitária de duas unidades distintas, e após examinar problemas específicos criando experimentos de pesquisa - entre eles a fala egocêntrica e a formação de conceitos -, Vigotski encerrou seu capítulo sobre "Pensamento e palavra" afirmando que "A palavra (significativa ${ }^{2}$ ) é um microcosmo da consciência humana" (VIGOTSKI, 2001b, 486). Torna-se evidente, ao longo de seu trabalho teórico, que sua busca por uma compreensão da consciência humana atravessa e distingue toda a sua obra.

Apesar dos inúmeros debates que o conceito de "significado (da palavra)" gerou - seja no que diz respeito às traduções dos termos na língua russa; seja nas considerações de possíveis significados circunstritos a uma palavra em si (falada, escrita, dicionarizada, etc.); seja em relação a um processo de significação mais abrangente como a produção de sentidos em instâncias dialógicas -, o fato é que a proposta dessa unidade condensa múltiplas relações entre pensamento e fala em suas dimensões histórica, cultural e individual. Em meio a tantas discussões, surgiu como uma unidade analítica viável e desafiadora, refletindo e refratando relações, condições e dinâmicas sociais. Esse gesto teórico-conceitual, que se percebe emergindo dos debates da época (e continua gerando debates acirrados ao longo de décadas), pode ser considerado uma contribuição metodológica de extrema relevância. Vigotski anunciou novas questões, abriu caminhos, sugeriu algumas formas de enfrentar os problemas e apontou elementos que sustentariam a realização posterior de trabalhos investigativos.

Assim, prosseguindo nas questões sobre o sentido e a unidade de análise, trazemos à tona um conceito que vem adquirindo grande repercussão, provocando debates e inspirando pesquisas renovadas sobre o desenvolvimento infantil: perezhivanie (FLEER; GONZALEZ REY; VERESOV, 2017; MIND, CULTURE AND ACTIVITY, 2016; MESHCHERYAKOV, 2010; TOASSA; SOUZA, 2010; VERESOV; FLEER, 2016). Vamos nos concentrar neste conceito, examinando e explorando seu potencial como uma unidade significativa de análise para estudos sobre o desenvolvimento infantil.

Segundo Vigotski (1994c), perezhivanie condensa as particularidades do ambiente - a representação dos elementos externos - e as particularidades da personalidade - como a pessoa

\footnotetext{
${ }^{2} \mathrm{Na}$ tradução do texto em português encontramos: "a palavra consciente é o microcosmo da consciência humana". No entanto, cotejando com outras traduções em outras línguas, optamos por referir à "palavra significativa" como microcosmo da consciência humana. 
vive, sente, significa; configura-se como uma união indivisível das dimensões individual e social, subjetiva e objetiva, cognitiva e emocional. Esta unidade permite explicar como as ações da criança afetam ou produzem efeitos no meio ambiente e este, por sua vez, torna-se fonte de desenvolvimento psíquico. Suas descobertas mostram que o ambiente se expande conforme a criança se desenvolve; que a criança expande sua experiência a partir dos novos elementos a que tem acesso. Isso significa que a análise do meio só é possível indiretamente pelos gestos da criança, pelo significado atribuído a cada vivência, pela forma como ela se relaciona afetivamente com uma determinada situação ou atividade.

Vigotski argumentou que uma abordagem científica do assunto deveria ter como objetivo formular as "leis do desenvolvimento". Embora essa maneira de formular suas ideias possa soar, hoje, um tanto determinista, deve-se levar em conta a base dialética e materialista de seu pensamento, bem como buscar métodos de pesquisa que deem conta de dimensões como integralidade, heterogeneidade e plasticidade na consideração do desenvolvimento humano. Assim, ele advogou em favor de uma análise qualitativa e intersistêmica que deveria considerar a unidade complexa dos diferentes aspectos determinantes dos processos de desenvolvimento; o movimento histórico de (trans)formação das funções psíquicas; as lacunas, os diversos ritmos e trajetórias desses processos; o surgimento de novas formações psicológicas - relacionadas com a sociedade; e a existência de períodos ótimos para o desenvolvimento da personalidade.

Partindo dessas premissas, a proposta analítica está enraizada na escolha de uma unidade de análise pelo pesquisador. Seu método trouxe à tona uma alternativa investigativa para a decomposição de fenômenos em elementos, utilizada por várias ciências, incluindo a psicologia. Ciente das desvantagens desta última, Vigotski considerou um conjunto complexo de fatores orgânicos e sociais, biológicos e culturais, todos implicados no desenvolvimento infantil.

Assim, ao assumir a relação imbricada criança-ambiente, um dos grandes esforços pode ser esboçar os procedimentos metodológicos e analíticos que permitam estudar a influência multidimensional do ambiente no desenvolvimento psíquico infantil, considerando a dinâmica e as condições que compõem sua configuração, sem desconsiderar o papel das peculiaridades da criança. Nesse sentido, perezhivanie surge como unidade de análise, permitindo-nos compreender como a criança atribui sentido às relações sociais que vivencia.

A análise da relação criança-meio ambiente, do ponto de vista da perezhivanie, orienta a pesquisa para os modos de participação e significação da criança nas práticas e relações sociais. Amplia, assim, a forma como o desenvolvimento infantil é concebido, considerado no 
contexto das condições concretas, das contradições sociais, das relações interpessoais, das dinâmicas interativas, das demandas sociais e dos processos de significação.

O significado da palavra e a perezhivanie constituem exemplos poderosos de como Vigotski concebia as possibilidades de estudar a consciência humana: tais unidades de análise indicam tentativas de configurar unidades dentro da totalidade, isto é, partes significativas de um todo dinâmico, levando em consideração um complexo de múltiplas determinações históricas. Múltiplas determinações significam aqui a gênese histórica, mas também o engendramento de novas formas de ser humano, de ser consciente.

A análise histórico-genética implica, portanto, uma compreensão de como a criança atua/opera de modo a provocar o desenvolvimento por meio de uma intervenção pedagógica intencionalmente orientada. Aqui reside a proposta de Vigotski de construção do método investigativo, em processo, em movimento. E aqui também reside a extrema importância desse processo investigativo relacionado ao trabalho educacional.

No trabalho investigativo, o prisma e o olhar analíticos exigem uma atitude de suspensão e desconfiança em relação ao que parece óbvio ou imediatamente evidente. Vigotski alerta para a importância de ler as entrelinhas, de procurar o que está por trás, de desvendar o que fica escondido, de prestar atenção aos pequenos detalhes:

O zoólogo reconstrói um esqueleto inteiro a partir de um fragmento insignificante de osso de algum animal escavado e até mesmo uma imagem de sua vida. Uma moeda antiga, sem nenhum valor como moeda, frequentemente revela ao arqueólogo um problema histórico complexo. O historiador, ao decifrar hieróglifos gravados em uma pedra, penetra nas profundezas de eras desaparecidas. O médico diagnostica a doença a partir de sintomas insignificantes. Só nos últimos anos a psicologia superou o medo da apreciação diária dos fenômenos e aprendeu por meio de minúcias insignificantes. (VIGOTSKI, 1995, p. 64, nossa tradução).

A perspectiva investigativa de Vigotski em relação ao desenvolvimento humano mostrase extremamente fértil em relação ao trabalho de pesquisa contemporâneo, entrando em diálogo e estabelecendo uma base sólida para o aprimoramento de análises microgenéticas, estudos etnográficos, análises de discurso, paradigmas indiciários e muitos outros campos e abordagens.

\section{Repercussões e implicações das ideias de Vigotski: Pesquisa contemporânea em educação}

$\mathrm{Na}$ introdução da primeira tradução para o inglês de Pensamento e linguagem, de Vigotski, Jerome Bruner observou que "a concepção de desenvolvimento de Vigotski era, ao mesmo tempo, uma teoria da Educação" (BRUNER, 1962, p. V). Mais tarde, no prólogo do primeiro volume de Collected Works (RIBER E\& CARTON, 1987), que incluía Pensamento e Linguagem, Bruner admitiu que, na época, ele "não conhecia nem a metade" do que imaginava 
saber sobre Vigotski. E expandiu: "Pois 'educação' implica, para Vigotski, não apenas o desenvolvimento do potencial do indivíduo, mas a expressão histórica e a expansão da cultura humana da qual o Homem surge" (BRUNER, 1987, p. 1-2; também MOLL, 1990, p. 1).

O envolvimento mais vívido e o compromisso genuíno de Vigotski com a educação em tempos revolucionários certamente tornou possível a elaboração de uma teoria inspiradora da atividade humana. Sua vivência e amplo diálogo com tantos interlocutores em diferentes áreas do conhecimento contribuíram para o desenvolvimento de um processo inovador de construção teórica. Ainda reverberam fortes repercussões de sua forma de conceber processos de investigação e intervenção em psicologia e educação.

Desde a década de 1980, uma enorme quantidade de pesquisas explorou e expandiu as propostas de Vigotski, sendo o Brasil um dos países da América Latina que mais contribuíram para os estudos e debates, especialmente no campo da educação. No final da década de 1970, a América Latina estava imersa em tempos políticos agitados e o Brasil vivia os últimos anos de ditadura. Pensadores e educadores brasileiros buscaram teorias alternativas consistentes que pudessem contribuir para a compreensão das condições de mudança do desenvolvimento humano e do processo educacional. Quando as ideias de Vigotski chegaram ao Brasil, caíram em terreno fecundo já fertilizado pelas reflexões de Paulo Freire sobre a educação e a elaboração social e política da consciência. Ambos os autores compartilhavam princípios e suposições marxistas.

No que diz respeito à questão do método, muitos autores têm comentado a obra de Vigotski, traçando o desenvolvimento de suas elaborações teóricas e metodológicas, descrevendo e comentando princípios e processos, destacando seus principais argumentos e contribuições especiais para os campos da psicologia e da educação (BERNARDES, 2010; DAVYDOV; RADZICKOWSKII, 1985; KOZULIN, 1990; LEONTIEV, 1997; VAN DER VEER; VALSINER, 1991; VERESOV, 1999; WERTSCH, 1985a e 1985b; WERTSCH; TULVISTE，1992; YAROCHEVSKI，1989; YAROSHEVSKY; GURGENIDZE， 1997; ZANELLA et al. 2007). Alguns discutiram especificamente o valor metodológico e os desafios de sua noção de unidade de análise (MATUSOV, 2007; TOOMELA, 2017; VAN DER VEER, 2001; ZINCHENKO, 1985).

É no campo da educação, no entanto, que as ideias de Vigotski tornam-se mais profundamente enraizadas e ramificadas ao mesmo tempo. Seus princípios teóricos e metodológicos têm afetado diretamente a pesquisa em educação, ao mesmo tempo ancorando e conduzindo a algumas ousadas formas de intervenção e investigação. Podemos perguntar: Quais são ou como se configuram os objetos de investigação na contemporaneidade? Como 
eles tem sido abordados atualmente? Conforme inscrito nos próprios modos de investigação de Vigotski, é fundamental prestar atenção às condições concretas do desenvolvimento humano, relacionado à elaboração histórica da consciência social/individual.

Muitos autores têm trabalhado e chamado a atenção para objetos específicos de estudo em contextos e políticas de educação formal, educação especial e inclusiva e educação em comunidades diversas e grupos minoritários, com foco nas relações de ensino-aprendizagem e discutindo meios e modos de apropriação da cultura em participação com as práticas sociais.

Entre aqueles cujo trabalho investigativo foi compartilhado por meio da produção acadêmica, podemos citar, desde o final dos anos 1970 até os anos 1980 - após as primeiras traduções e a disseminação das ideias de Vigotski na Europa e nas Américas -, as tentativas de Wertsch, Stone, Rogoff e Hickmann de desenvolver o que chamaram de análise microgenética, derivada das noções de Vigotski de internalização e zona de desenvolvimento proximal (ROGOFF; WERTSCH, 1984; WERTSCH; HICKMANN, 1987; WERTSCH; STONE, 1978). Naquela época, Scribner e Cole também desenvolviam pesquisas sobre cultura e cognição, tentando entender muitas maneiras diferentes de pensar e aprender por meio de uma abordagem Vigotskiana (COLE, 1985; NEWMAN; GRIFFIN; COLE, 1989; SCRIBNER; COLE, 1981).

Estudos interdisciplinares nas interseções da psicologia, antropologia e educação, envolvendo aprendizagem em comunidades de prática, comunidades escolares e processos de escolarização, também se desenvolveram, com foco em práticas culturais e de ensino com crianças de classe baixa, marginais ou imigrantes, indígenas ou pessoas rurais (ROGOFF; LAVE, 1984; ROGOFFF, 1990; CANDELA; ROCKWELL, 1991; HEDEGAARD, 1990, 1996; LAVE, 1996; LAVE; WENGER, 1991; PARADISE, 1991; ROCKWELL, 1999; ROCKWELL; MERCADO, 1999).

De fato, foi durante a década de 1990 que a produção de conhecimento na perspectiva histórico-cultural experimentou uma grande expansão. Um número incontável de artigos, livros e coleções de textos, contendo relatos de pesquisas e discussão de conceitos e suas implicações práticas em contextos educacionais, adquiriram visibilidade em reuniões científicas e publicações. Durante esta década, as novas configurações políticas mundiais, os processos de globalização e o desenvolvimento de tecnologias criaram novas condições de produção, acesso e intercâmbio acadêmico, que se expandiram e se tornaram ainda mais dinâmicas ao longo do século XXI. Pelo menos três periódicos divulgaram as principais ideias e pesquisas de Vigotski: Mind, Culture and Activity (originado em 1994); Cultura e Psicologia (originado em 1995); e o Journal of Russian \& East European Psychology, de 1992, anteriormente intitulado Soviet Psychology (1966-1991). 
Essas condições não apenas permitiram, mas aumentaram o acesso às obras e ideias desenvolvidas pelos colegas e seguidores de Vigotski - incluindo métodos de ensino e didática, como a proposta de Davydov de ensino desenvolvimental (DAVYDOV, 1988, 1995, 1999) cujas novas contribuições se relacionam com a ampliação da ideia de situações experimentais de ensino (CHAIKLIN, 2003; LIBÂNEO; FREITAS, 2013; LONGAREZI; PUENTES, 2013).

Os volumes editados por Moll (1990), Góes e Pino (1991), Daniels (1993), Wertsch, Del Rio e Alvarez (1995), Chaiklin e Lave (1996), Moro, Schneuwly e Brossard (1997), Hedegaard e Lompscher (1999), Clot (1999B, 2012), Chaiklin (2001), Kozulin et al. (2003), Daniels, Cole e Wertsch (2007), Daniels e Hedegaard (2011), Davis et al. (2015), Yasnitsky, Van der Veer e Ferrari (2014) dão testemunho do amplo e representativo grupo de educadores e pesquisadores que atuam na área e incluem estudos sobre as mais diversas condições de desenvolvimento.

É importante destacar ainda as obras de autores que, mais recentemente, têm comentado explicitamente os objetivos políticos e os compromissos éticos implícitos nas propostas teóricometodológicas de Vigotski, explorando e enfatizando a força e a pertinência das propostas para a pesquisa contemporânea e o trabalho educacional (DELARI JUNIOR, 2015; STETSENKO, 2016, 2017).

Seguindo uma tendência mundial, a perspectiva da pesquisa histórico-cultural se difundiu amplamente nas últimas décadas no Brasil. Grupos de pesquisa sediados em universidades e centros de investigação em todo o país adotaram os princípios e métodos Vigotskianos e lançaram diferentes tipos de projetos com o objetivo de compreender e explicar as condições de vida e ensino-aprendizagem de famílias, escolas e outras instituições onde ocorre educação formal ou não formal (DUARTE, 1996; GÓES, 1996; OLIVEIRA, 1993; SMOLKA, 1988, 2000; SMOLKA; GÓES, 1993). Eles estavam comprometidos, também, com a produção de mudanças nesses ambientes sociais. A grande variedade de objetos e focos de estudo, bem como o número de pesquisadores envolvidos nesse processo, torna impossível enumerá-los. Ainda assim, alguns exemplos podem ser citados, tais como: estudos sobre práticas pedagógicas e formas de ensino e aprendizagem, políticas públicas e estaduais de educação, alfabetização e aquisição de conhecimentos (AGUIAR, 2006; DUARTE, 1996; FACCI; TULESKI; BARROCO，2009; MORTIMER，2000; SFORNI; BERNARDES; MOURA, 2003; SMOLKA; NOGUEIRA, 2013), educação especial e educação inclusiva (GÓES; LAPLANE, 2004; PADILHA; OLIVEIRA, 2013), educação infantil, acolhimento familiar e adoção (ROSSETTI- FERREIRA, et al., 2003; ROSSETTI-FERREIRA, et al., 2008), e o uso de ferramentas técnico-semióticas (FREITAS, 2009), entre muitas outras. 
Em todo o mundo, pesquisadores discutiram criativamente outras tendências teóricas e metodológicas, perspectivas e técnicas derivadas da sociologia, psicologia social, linguística, antropologia, filosofia e ciência política, produzindo novas sínteses (como em ÁLVAREZ; DEL RÍO, 2007; CLOT, 1999a; DEL RÍO, 2002, 2007; ENGESTROM, 2007; ENGESTROM, SANNINO; VIRKKUNEN, 2014; GONZÁLEZ REY, 2016a, 2016b). Os primeiros grupos de pesquisa consolidados tornaram-se o locus de uma nova geração de pesquisadores que continuaram a tarefa de desenvolver novas agendas de pesquisa (VERESOV; SMOLKA; PARADISE, 2013). Eles passaram a incluir problemas emergentes em consonância com os movimentos históricos, políticos e teóricos que impactaram as práticas sociais e educacionais.

A perspectiva histórico-cultural tem sólida presença mundial. No Brasil, nas últimas décadas, diferentes fontes que contemplam os conceitos e ideias de Vigotski integram os currículos de diversos cursos universitários (Psicologia, Pedagogia, Educação Especial), além de documentos educacionais oficiais e diretrizes e políticas curriculares de educação básica e especial. Os resultados da pesquisa frequentemente fundamentam debates, discussões e a elaboração de políticas nos níveis estadual, municipal ou nacional. Mas, apesar dessa aparente adesão à perspectiva, este tem sido, na realidade, um locus de intensa luta. Luta não apenas para proclamar "educação para todos" ou "todos pela educação", mas por um trabalho efetivo na transformação e criação de novas condições concretas de existência, diminuindo a extrema desigualdade das condições de vida humana.

Se, como afirmava Vigotski, as relações sociais são o principal fator determinante da atividade humana individual, a pesquisa sobre o desenvolvimento humano e a educação deve levar em conta a dinâmica dessas relações e condições. Concebida como um processo interveniente e (trans)formador, a atividade de pesquisa exige uma abordagem sensível às mais diversas formas de agir, relacionar-se, apropriando-se de ferramentas e símbolos e produzindo sentido, ou seja, participando de práticas sociais. A educação formal, historicamente instituída com o objetivo de orientar e criar modos de participação individual e coletiva na cultura, tornase, assim, um locus privilegiado de estudo das (condições de) desenvolvimento humano individual, social, histórico.

Como observa Stetsenko $(2016,2017)$, Vigotski pensou em uma nova psicologia voltada para a reconstrução da sociedade com base nos princípios da justiça, solidariedade e igualdade social, implicando, assim, uma ética sociopolítica que desafia a ideologia da adaptação associada à premissa de passividade, desigualdade inata e controle social. Nessa perspectiva, o método pode ser entendido como meio/modo de organização da atividade 
teórico-prática, articulado à construção histórica da consciência coletiva/individual visando à transformação social.

Os princípios do materialismo histórico-dialético podem ser reconhecidos nos fundamentos dos conceitos formulados por Vigotski, bem como nos instrumentos analíticos em que se apoia para investigar o processo de desenvolvimento humano e definir seu objeto de estudo e método. Neste contexto, a adequação entre objeto e método significa fazer escolhas e justificá-las pelos princípios que ele assume.

As considerações metodológicas de Vigotski nos impelem a compreender o caráter (trans)formador da educação como prática sócio-histórica e a enfatizar as dimensões éticas e políticas da produção de conhecimento. Estas estão intimamente relacionadas ao compromisso social consignado no ato de pesquisar, que assume uma abordagem qualitativa, não neutra, sustentada por problemas sociais e carregada pelo compromisso de criar condições de desenvolvimento cultural - especialmente por meio da educação - dos grupos sujeitos a processos de exclusão em razão da desigualdade social, diversidade cultural, deficiência ou outras condições. Particularmente no campo da educação, essa perspectiva teóricometodológica é promissora no apoio a pesquisas que vinculem a problemática do desenvolvimento humano às práticas educacionais. Ela fornece os meios para investigar e teorizar sobre o desenvolvimento e a vida social, explorando o estatuto e o significado das práticas educacionais transformadoras.

\section{Referências}

AGUIAR, W. M. J. (Ed.). Sentidos e significados do professor na perspectiva sóciohistórica: relatos de pesquisa. São Paulo: Casa do Psicólogo, 2006.

ÁLVAREZ, A.; DEL RÍO, P. The zone of proximal development: Inside and outside. In: DANIELS, H., COLE, M.; WERTSCH, J. V. (Eds.). The Cambridge companion to Vygotsky. Cambridge: Cambridge University Press, 2007. p. 276-303.

BERNARDES, M. E. M. O método de investigação na psicologia histórico-cultural e a pesquisa sobre o psiquismo humano. Revista Psicologia Política, v. 10, n. 20, p. 297-313, 2010.

BRUNER, J. Introduction. In: VYGOTSKY, L. S. Thought and language. Cambridge: MIT Press, 1962.

BRUNER, J. Prologue to the English edition. In: RIEBER, R. W.; CARTON, A. S. (Eds.). The collected works of L. S. Vygotsky: Problems of general psychology. New York: Plenum Press, 1987. p. 35-283.v. 1. 
CANDELA, A.; ROCKWELL, E. Construcción social del conocimiento en el aula: Un enfoque etnográfico. Infancia y Aprendizaje, v. 14 n. 55, p. 3-11, 1991.

CHAIKLIN, S. The zone of proximal development in Vigotski's analysis of learning and instruction. In: KOZULIN, A., et al. (Eds.). Vygotsky's educational theory in cultural context. Cambridge University Press, 2003.

CHAIKLIN, S. (Ed.). The theory and practice of cultural-historical psychology. Aarhus: Aarhus University Press, 2001.

CHAIKLIN, S.; LAVE, J. (Eds.). Understanding practice. Cambridge, U.K.: Cambridge University Press, 1996.

CLOT, Y. La function psychologique du travail. Paris: Presses Universitaires de France, 1999a.

CLOT, Y. (Ed.). Avec Vygotski. Paris, France: La Dispute, 1999 b.

CLOT, Y. (Ed.). Vygotski maintenant. Paris, France: La Dispute, 2012.

COLE, M. The zone of proximal development: Where culture and cognition create each other. In: WERTSCH, J. V. (Ed.). Culture, communication and cognition: Vygotskian perspectives. Cambridge, U.K.: Cambridge University Press, 1985. p. 146-161.

DANIELS, H. (Ed.). Charting the agenda: Educational activity after Vigotski. London, U.K.: Routledge, 1993.

DANIELS, H.; COLE, M.; WERTSCH, J. V. (Eds.). The Cambridge companion to Vygotsky. Cambridge, U.K.: Cambridge University Press, 2007.

DANIELS, H.; HEDEGAARD, M. (Eds.). Vygotsky and special needs education rethinking support for children and schools. London, U.K.: Continuum International, 2011.

DAVIS, S. et al. (Eds.). Dramatic interactions in education: Vygotskian and sociocultural approaches to drama, education and research. London, U.K.: Bloomsbury, 2015.

DAVYDOV, V. V. La enseñanza escolar y el desarrollo psíquico. Moscou: Progresso, 1988.

DAVYDOV, V. V. The influence of L. S. Vygotsky on education theory, research and practice. Educational Researcher, v. 24, n. 3, p. 12-21, 1995.

DAVYDOV, V. V. What is real learning activity? In: HEDEGAARD, M.; LOMPSTER, J. (Eds.). Learning activity and development. Aarhus: Aarhus University Press, 1999.

DAVYDOV, V. V.; RADZIKHOVSKII, L. A. Vygotsky's theory and the activity-oriented approach in psychology. In: WERTSCH, J. V. (Ed.), Culture, communication, and cognition. New York: Cambridge University Press, 1985. p. 35-65.

DEL RÍO, P. The external brain: Eco-cultural roots of distancing and mediation. Culture \& Psychology, v. 8, n. 2, p. 233-265, 2002.

DEL RÍO, P. Educación y evolución humana: contribución al debate ¿Qué teorías necesitamos en educación? Cultura y Educación, v. 19, n. 3, p. 231-241, 2007.

DELARI JUNIOR, A. Questões de método em Vigotski: busca da verdade e caminhos da cognição. In: TULESKI, S. C.; CHAVES, M.; LEITE, H. A. (Eds.). Materialismo histórico- 
dialético como fundamento da psicologia histórico-cultural: método e metodologia de pesquisa. Maringá: Eduem, 2015. p. 43-82.

DUARTE, N. Educação escolar, teoria do cotidiano e a escola de Vigotski. Campinas: Autores Associados, 1996.

ENGESTROM, Y. Putting Vygotsky to work: The change laboratory as an applicationof double stimulation. In: DANIELS, H.; COLE, M.; WERTSCH, J. V. (Eds.). The Cambridge companion to Vigotski. Cambridge, U.K.: Cambridge University Press, 2007. p. 363-382.

ENGESTROM, Y.; SANNINO, A.; VIRKKUNEN, J. On the methodological demands of formative interventions. Mind, Culture, and Activity, v. 21, n. 2, p. 118-128, 2014.

FACCI, M. G. D.; TULESKI, S. C.; BARROCO, M. S. (Eds.). Escola de Vigotski: contribuições para a psicologia e a educação. Maringá: Eduem, 2009.

FleEr, M.; GONZAlEZ REY, F.; VERESOV, N. (Eds.). Perzhivanie, emotions and subjectivity: Advancing Vigotski’s legacy. Singapore: Springer, 2017.

FREITAS, M. T. A. Cibercultura e formação de professores. Belo Horizonte: Autêntica, 2009.

GÓES, M. C. R., de. Linguagem, surdez e educação. Campinas: Autores Associados, 1996.

GÓES, M. C. R., de; LAPLANE, A. L. F., de. (Eds.). Políticas e práticas de educaçãoinclusiva. Campinas: Autores Associados, 2004.

GÓES, M. C. R., de; PINO, A. Apresentação. Cadernos Cedes, v. 24, p. 5-9, 1991.

GONZÁLEZ REY, F. Advancing the topics of social reality, culture, and subjectivity from a cultural-historical standpoint: Moments, paths, and contradictions. Journal of Theoretical and Philosophical Psychology, v. 36, n. 3, p. 175-189, 2016a.

GONZÁLEZ REY, F. Marxismo, subjetividad y psicología cultural-histórica: avanzando sobre un legado inconcluso. Teoría y Critica de la Psicología, v. 7, p. 40-55, 2016 b.

HEDEGAARD, M. The zone of proximal development as basis for instruction. In: MOLL, L. C. (Ed.). Vygotsky and education. Cambridge, U.K.: Cambridge University Press, 1990. p. 349371.

HEDEGAARD, M. How instruction influences children's concepts of evolution. Mind, Culture, and Activity, v. 3, n. 1, p. 11-24, 1996.

HEDEGAARD, M.; LOMPSCHER, J. (Eds.). Learning activity and development. Aarhus: Aarhus University Press, 1999.

KOZULIN, A. Vygotsky's psychology: A biography of ideas. Cambridge, MA: Harvard University Press, 1990.

KOZULIN, A. et al. (Eds.). Vygotsky's educational theory in cultural context. Cambridge, U.K.: Cambridge University Press, 2003.

LAVE, J. Teaching, as learning, in practice. Mind, Culture, and Activity, v. 3, n. 3, p. 149-164, 1996. 
LAVE, J.; WENGER, E. Situated learning: Legitimate peripheral participation. Cambridge, U.K.: Cambridge University Press, 1991.

LEONTIEV, A. N. On Vygotsky's creative development. In: RIEBER, R. W.; WOLLOCK, J. (Eds.). The collected works of L. S. Vygotsky: Problems of the theory and history of psychology. New York: Plenum Press, 1997. p. 9-34. v. 3.

LEONTIEV, A. N. The development of mind. In: CC-SA, Marxists Internet Archive. Selected works of Aleksei Nikolaevich Leontyev, 2009.

LIBÂNEO, J. C.; FREITAS, R. A. M. M. Vasily Vasilyevich Davydov: a escola e a formação do pensamento teórico-científico. In: LONGAREZI, A. M.; PUENTES, R. V. (Eds.). Ensino desenvolvimental: vida, pensamento e obra dos principais representantes russos. Uberlândia: EDUFU, 2013.

LONGAREZI, A. M.; PUENTES, R. V. (Eds.). Ensino desenvolvimental: vida, pensamento e obra dos principais representantes russos. Uberlândia: EDUFU, 2013.

LURIA, A. The development of writing in the child. Soviet Psychology, v. 16, n. 2, p. 65-114, 1977.

MARX, K. Para a crítica da economia política. In: GIANNOTTI, J. A. (Ed.). Marx: vida e obra. São Paulo: Nova Cultural, 1999.

MARX, K.; ENGELS, F. A ideologia alemã (Feuerbach). São Paulo: Hucitec, 1987.

MATUSOV, E. In search of "the appropriate" unit of analysis for sociocultural research. Culture \& Psychology, v. 13, n. 3, p. 307-333, 2007.

MESHCHERYAKOV, B. G. Views of L. S. Vygotsky at the science about children's development. Psicologia USP, v. 21, n. 4, p. 703-726, 2010.

MIND, CULTURE, AND ACTIVITY: An International Journal. Ed. especial: Symposium on Perezhivanie, v. 23, n. 4, 2016.

MOLL, L. C. (Ed.). Vygotsky and education: Instructional implications and applications of sociohistorical psychology. Cambridge, U.K.: Cambridge University Press, 1990.

MORO, C.; SCHNEUWLY, B.; BROSSARD, M. (Eds.). Outils et signes. Perspectives actuelles de la théorie de Vygotski. Bern: Peter Lang, 1997.

MORTIMER, E. F. Linguagem e formação de conceitos no ensino de ciências. Belo Horizonte: UFMG, 2000.

NEWMAN, D.; GRIFFIN, P.; COLE, M. The construction zone: Working for the cognitive change in school. Cambridge, U.K.: Cambridge University Press, 1989.

OLIVEIRA, M. K., de. Vigotski: aprendizado e desenvolvimento. Um processo sóciohistórico. São Paulo: Scipione, 1993.

PADILHA, A. M. L.; OLIVEIRA, I. M., de. (Eds.). Educação para todos: as muitas faces da inclusão escolar. Campinas: Papirus, 2013.

PARADISE, R. Cultural knowledge in the classroom: Indigenous children and their orientation towards observation. Infancia y Aprendizaje, v. 14, n. 55, p. 73-85, 1991. 
PINO, A. The social and the cultural in Vygotsky's work. Educação \& Sociedade, v. 71, p. 45$78,2000$.

RIEBER, R. W. (Ed.). The collected works of L. S. Vygotsky: The history of the development of higher mental functions. New York: Plenum Press, 1997. v. 4.

RIEBER, R. W.; CARTON, A. S. (Eds.). The collected works of L. S. Vygotsky: The fundamentals of defectology (abnormal psychology and learning disabilities). New York: Plenum Press, 1993. v. 2.

RIEBER, R. W.; CARTON, A. S. (Eds.). The collected works of L. S. Vygotsky: Problems of the theory and history of psychology. New York: Plenum Press, 1997. v. 3.

ROCKWELL, E. Historical consciousness and critical thinking: Reflections on an indigenous movement. Educational Foundations, v. 13, n. 3, p. 23-39, 1999.

ROCKWELL, E.; MERCADO, R. La escuela, lugar del trabajo docente. México: DIECINVESTAV-IPN, 1999.

ROGOFF, B. Apprenticeship in thinking: Cognitive development in social context. New York: Oxford University Press, 1990.

ROGOFF, B.; LAVE, J. (Eds.). Everyday cognition: Its development in social contexts. Cambridge, MA: Harvard University Press, 1984.

ROGOFF, B.; WERTSCH, J. V. Children's learning in the "zone of proximal development." New Directions for Child Development, San Francisco, v. 23, 1984.

ROSSETTI-FERREIRA, M. C., et al. (Eds.). Rede de significações e o estudo do desenvolvimento humano. Porto Alegre: Artmed, 2003.

ROSSETTI-FERREIRA, M. C. et al. Approaching adoption and foster care in Brazil. Adoption Quarterly, v. 11, n. 1, p. 67-77, 2008.

SCRIBNER, S.; COLE, M. The psychology of literacy. Cambridge, MA: Harvard University Press, 1981.

SFORNI, M. S. F.; BERNARDES, M. E. M.; MOURA, M. O., de. A organização do pensamento e a aprendizagem de conceitos na atividade de ensino. Psicologia (Braga), v. 1, p. 45- 57, 2003.

SMOLKA, A. L. B. A criança na fase inicial da escrita: a alfabetização como processo discursivo. São Paulo: Cortez, 1988.

SMOLKA, A. L. B. O (im)próprio e o (im)pertinente na apropriação das práticas sociais. Cadernos Cedes, v. 50, p. 26-40, 2000.

SMOLKA, A. L. B.; GÓES, M. C. R., de. (Eds.). A linguagem e o outro no espaço escolar: Vigotski e a construção do conhecimento. Campinas: Papirus, 1993.

SMOLKA, A. L. B.; NOGUEIRA, A. L. H. (Eds.). Estudos na perspectiva de Vigotski:gênese e emergência das funções psicológicas. Campinas: Mercado de Letras, 2013.

STETSENKO, A. Vygotsky's theory of method and philosophy of practice: Implications for trans/formative methodology. Educação, Porto Alegre, v. 39, Suplemento especial, s32-s41, 2016. 
STETSENKO, A. The transformative mind: Expanding Vygotsky's approach to development and education. New York: Cambridge University Press, 2017.

TOASSA, G.; SOUZA, M. P. R. As vivências: questões de tradução, sentidos e fontes epistemológicas no legado de Vigotski. Psicologia USP, São Paulo, v. 21, n. 4, p. 757-779, 2010 .

TOOMELA, A. Methodology of cultural-historical psychology. In: YASNITSKY, A.; VAN DER VEER, R.; FERRARI, M. (Eds.). The Cambridge handbook of cultural-historical psychology. Cambridge, U.K.: Cambridge University Press, 2017. p. 101-124.

VAN DER VEER, R. The idea of unit of analysis: Vygotsky's contribution. In: CHAIKLIN, S. (Ed.). The theory and practice of cultural-historical psychology. Aarhus: Aarhus University Press, 2001.

VAN DER VEER, R.; VALSINER, J. Vygotsky: uma síntese. São Paulo: Edições Loyola, 1991.

VAN DER VEER, R.; VALSINER, J. (Eds.). The Vygotsky reader. Oxford: Blackwell, 1994.

VERESOV, N. (Ed.). Undiscovered Vygotsky. Frankfurt: Peter Lang, 1999.

VERESOV, N.; FLEER, M. Perezhivanie as a theoretical concept for researching young children's development. Mind, Culture, and Activity. Special issue: Symposium on Perezhivanie, v. 23, n. 4, p. 56-66, 2016.

VERESOV, N. N.; SMOLKA A. L.; PARADISE R. Expanding the cultural historical theory: Fourth generation is coming? Cultural-Historical Psychology, v. 3, p. 46-55, 2013.

VIGOTSKI, L.S. Formação social da mente. São Paulo: Martins Fontes, 1984.

VIGOTSKI, L. S. Teoria e método em psicologia. São Paulo: Martins Fontes, 1996.

VIGOTSKI, L. S. A Tragédia de Hamlet, Príncipe da Dinamarca. São Paulo: Martins Fontes, 1999a.

VIGOTSKI, L. S. Psicologia da arte. São Paulo: Martins Fontes, 1999 b.

VIGOTSKI, L. S. Manuscrito de 1929. Educação \& Sociedade, ano XXI, n. 71, jul. 2000. Disponível em: www.scielo.br/pdf/es/v21n71/a02v2171.pdf

VIGOTSKI, L. S. Psicologia Pedagógica. São Paulo: Martins Fontes, 2001a.

VIGOTSKI, L.S. A construção do pensamento e da linguagem. São Paulo: Martins Fontes, $2001 b$.

VYGOTSKI, L. S. Problemas del desarrollo de la psique. Obras Escogidas. Vol. III. Madrid: Visor Distribuiciones, S.A. 1995.

VYGOTSKI, L. S. Psicología infantil. Obras Escogidas. Vol.IV. Madrid: Visor Distribuiciones, S.A.1996.

VYGOTSKI, L. S. Fundamentos de Defectologia. Obras Escogidas. Vol.V. Madrid: Visor Distribuiciones, S.A, 1997. 
VIGOTSKI, L. S. Sete aulas sobre os fundamentos da pedologia. Tradução de Zoia Prestes. Rio de Janeiro: E-papers, 2018.

VYGOTSKY, L. S.; LURIA, A. Tool and symbol in child development. In: VAN DER VEER, R.; VALSINER, J. (Eds.). The Vigotski reader. Oxford: Blackwell, 1994. p. 99-174.

WERTSCH, J. V. (Ed.). Culture, communication, and cognition. Cambridge: Cambridge University Press, 1985a.

WERTSCH, J. V. (Ed.). Vigotski and the social formation of mind. Cambridge, MA: Harvard University Press, 1985 b.

WERTSCH, J. V.; DEL RIO, P.; ALVAREZ, A. (Eds.). Sociocultural studies of mind. Cambridge, U.K.: Cambridge University Press, 1995.

WERTSCH, J. V.; HICKMANN, M. Problem solving in social interaction: A microge netic analysis. In: HICKMANN, M. (Ed.). Social and functional approaches to language and thought. Orlando: Academic Press, 1987. p. 251-266.

WERTSCH, J. V.; STONE, C. A. Microgenesis as a tool for developmental analysis. Quarterly Newsletter of the Laboratory of Comparative Human Development, v. 1, n. 1, p. 8-10, 1978.

WERTSCH, J. V.; TULVISTE, P. L. S. Vygotsky and contemporary developmental psychology. Developmental Psychology, v. 28, p. 548-557, 1992.

YAROCHEVSKI, M. G. Lev Vygotsky. Moscou: Progress Publishers, 1989.

YAROSHEVSKY, M. G.; GURGENIDZE, G. S. Epilogue. In: RIEBER, R. W.; WOLLOCK J. (Eds.). The collected works of L. S. Vygotsky: Problems of the theory and history of psychology. New York: Plenum Press, 1997. p. 345-369. v. 3.

YASNITSKY, A.; VAN DER VEER, R.; FERRARI, M. (Eds.). The Cambridge handbook of cultural-historical psychology. Cambridge, U.K.: Cambridge University Press, 2014.

ZANELLA, A. V. et al. Method issues in Vygotsky's texts: Contributions to psychology research. Psicologia \& Sociedade, v. 19, n. 2, p. 25-33, 2007.

ZINCHENKO, V. P. Vygotsky's ideas about units for the analysis of mind. In: WERTSCH, J. V. (Ed.). Culture, communication and cognition: Vygotskian perspectives. Cambridge: Cambridge University Press, 1985. p. 94-118.

\footnotetext{
i O texto foi publicado originalmente em inglês em Education. Oxford Research Encyclopedia of Education. 1 ed.: Oxford University Press, 2019. A tradução para o português foi realizada por Cristina Paixão Lopes com revisão das autoras.
} 